\title{
Generalized internal multiple imaging (GIMI) using Feynman-like diagrams
}

\author{
M. A. H. Zuberi and Tariq Alkhalifah \\ 4700 King Abdullah University of Science and Technology, Thuwal 23955-6900, Saudi Arabia. E-mail: mohammad.zuberi@kaust.edu.sa
}

Accepted 2013 December 29. Received 2013 December 26; in original form 2013 August 28

\begin{abstract}
S UM M A R Y
Single scattering events recorded in surface seismic data do not fully illuminate the subsurface structure, especially if it is complicated. In such cases, multiple internal scatterings (internal multiples) can help improve the illumination. We devise a generalized internal multiple imaging (GIMI) procedure that maps internal multiple energy to their true location with a relatively mild addition to the computational cost. GIMI theory relies heavily on seismic interferometry, which often involves cumbersome algebra, especially when one is dealing with high-order terms in the perturbation series. To make the derivations, and inference of the results easier, we introduce Feynman-like diagrams to represent different terms of the perturbation series (solution to the Lippman-Schwinger equation). The rules we define for the diagrams allow operations like convolution and cross-correlation in the series to be compressed in diagram form. The application of the theory to a double scattering example demonstrates the power of the method.
\end{abstract}

Key words: Interferometry; Body waves; Wave scattering and diffraction.

\section{INTRODUCTION}

In seismic wavefields recorded on Earth's surface, single scattering energy often registers as the strongest signal from which the information about location and strength of the subsurface scatterers can be extracted. In a typical surface seismic experiment the recording area does not enclose the medium which contains these scatterers, therefore, it could happen that the path of the wavefield after single scattering does not end up at the recording area. This is most likely to happen in the case of complicated velocity models. In other words, in the case of a complicated subsurface velocity it is highly likely that single scattering will not illuminate some regions in the subsurface. Multiple scattering can be invaluable in these situations, as either second-, third- or even higher order internal multiples might have a path that ends on the recording area. In addition to that, even when a region of the subsurface is illuminated by single scattering, imaging of multiple internal scatterings (internal multiples) will enhance the final image. In this study, we suggest a generalized internal multiple imaging (GIMI) method to image different orders of internal multiples. In this method, we can enhance the image for any given order of internal multiples. Other methods of imaging the internal multiples have been suggested, for example, recently Behura et al. (2012) proposed a method based on virtual source creation Wapenaar et al. (2012) using inverse scattering. That method images all orders of internal multiples simultaneously and does not allow selection of individual orders or internal multiples to be imaged, which means that imaging cost is the same whether we only need double scattering to image a particular velocity model or a very high-order multiple. Furthermore, this method requires muting beyond the direct arrivals from subsurface image point to the surface, which adds to the cost of application. In GIMI, the only additional cost is the cross-correlation of the data with itself $N-1$ times, where $N$ is the order of the Born scattering term to be imaged. Although there are savings in computational cost, GIMI involves crosstalk which the method suggested by Behura et al. (2012) does not have. As discussed later the amplitude of crosstalk is weaker than the actual image of scatterers, therefore, we just square the image to suppress it. Another method suggested by Malcolm et al. (2009) uses the illumination decomposition, in which certain types of scatterings are ignored. In our case, we do not make any assumptions on the type of internal scattering.

The only requirement for GIMI is that the path of the wave allows it to reach the recording area on the surface, and no requirements on the amplitude of wavefield, which could even be zero. This can be understood by considering an analogous situation in the convolution-based surface multiple creation Verschuur et al. (1992). Surface multiples can be created by convolving the surface seismic data with itself even if originally it did not have any energy. Then, these surface multiples can be imaged by using any of the methods for imaging the surface multiples, for example Berkhout \& Verschuur (1994), Shan (2003) and Zuberi \& Alkhalifah (2013a). The only requirement is that the true velocity model is such that the wave paths of these surface multiples end on the recording area, if not, then these would not be created by the 
convolution procedure in the first place. This means that in GIMI we are simultaneously creating and imaging the internal multiples, provided that they are kinematically allowed to be in the data. However, we ignore surface multiples in this study and focus on only internal multiples.

In GIMI, we rely on seismic interferometric equations, which involve integrals over a surface enclosing the volume of interest (region to be imaged). Although Sommerfeld radiation condition can be used to justify the use of boundary integrals with surface seismic data, in our case an approximation to the reciprocity theorem of cross-correlation type is being used which does not satisfy the Sommerfeld radiation condition $^{1}$ (Wapenaar \& Fokkema 2006). This means that we would have to contend with some errors, which include amplitude errors. Another, more important, source of error in the amplitude is the cross-correlation itself. Ideally, to get the amplitudes right one should use deconvolution, which would kinematically be equivalent to cross-correlation, but treat the amplitudes accurately with a proper division in the frequency domain. However, deconvolution of wavefields has its own problems and in this study instead of dealing with them we just ignore the amplitudes. GIMI also involves crosstalk, which will be explained below and we shall suggest a trivial method to suppress this noise. Synthetic examples at the end show the usefulness of GIMI in imaging a simple velocity model with a vertical reflector. We place a scatterer beside this vertical reflector to create double scattering. Then, we show that GIMI is able to image part of that vertical reflector while single scattering approach is not capable of doing that.

\section{QUANTUM SCATTERING THEORY IN SEISMICS}

From the blue colour of the sky to the three colours of quarks, there is a wide range of natural phenomena that can be analysed through the scattering of fields and particles. Therefore, scattering theory has been used and developed in many branches of pure and applied physics. To put things in perspective we shall take a quick look at the theories that have found their way into seismic applications. These can be put into two broad categories, namely, quantum mechanical scattering and quantum field theoretic (QFT) scattering.

First, let us consider the quantum mechanical scattering. The scattering potential can be determined from scattered-field data by using the inverse scattering theory. There are two main methods, Gelfand-Levitan method and Marchenko method (Chadan \& Sabatier 1977). Gelfand-Levitan method hasn't been used much in seismic applications, but Marchenko method has found it's way to applications in seismic. Aktosun \& Rose (2002) have used Marchenko method to focus a 1-D plasma wave at some desired location and Rose (2004) applied it to 1-D acoustic wave in layered media. This method was then developed for use in 1-D seismic applications by Broggini \& Snieder (2012) and extended to 2-D by Wapenaar et al. (2012). Wapenaar et al. (2012) used it to create virtual sources in the subsurface. Quantum mechanical scattering is based on the Lippman-Schwinger equation that is the integral form of the time-independent Schrodinger equation. An analogous Lippman-Schwinger equation for acoustic fields is used by Weglein et al. (2003), but they take a different route to inverse scattering. In their method Weglein et al. (2003) expand the velocity perturbation as a series in wavefield. The forward Born series is an expansion of wavefield in velocity perturbation, therefore the inverse series is called the inverse Born series. The terms of inverse Born series are similar to the transition matrix series Sakurai (1993) in quantum mechanics. This method also bears resemblance to the one suggested by Jost \& Kohn (1952) for calculating the potential from the partial waves Sakurai (1993).

Analogies to scattering in QFT are harder to find. One obvious reason is that the fields in QFT are operators that have to satisfy quantum commutation (or anti-commutation) relations and this makes the calculation of scattering cross-sections quite different. Another, perhaps more challenging, problem is that the scattering in QFT comes as a result of adding non-linear terms to the Lagrangian density of the fields Zee (2003). Whereas in seismic applications the wave equations and therefore the associated Lagrangian densities always remain linear. Nonetheless, if we restrict ourselves to the free-field (non-interacting) theory, we can find a relation. For example, the free propagator for Klein-Gordon field, with zero mass, is just the Green's function in a constant velocity acoustic medium. An arbitrary Fourier mode of the propagator is called a virtual particle when it is not on mass shell ${ }^{2}$ (Zee 2003). Ikelle \& Gangi (2007) obtained reflections (virtual reflections) by cross-correlating different parts of the data and identified them with virtual particles. Another development in QFT that can be used readily in seismic applications is the path integral formulation itself and indeed it has been used. Fishman et al. (1987) use a propagation operator in exponential form to advance the one-way acoustic wavefield in infinitesimal steps. The total movement of the wavefield is obtained by multiplying these propagation operators and integrating over all possible paths. The stationary phase principle ensures that only the path satisfying Fermat's principle remains. The path integral approach has also been used by Landa et al. (2006) to explicitly define summation along all possible paths in order to obtain the seismic image. Other than the path integral formalism, QFT also offers elegant notation for analysis of perturbation series, that is, the Feynman diagrams. A graphical representation has been used by Ikelle \& Gangi (2007) to depict reflections in a layered medium. Although these representations provide a physical picture of the ray paths of reflected and refracted waves, they become complicated as the medium becomes complicated and/or order of multiples gets higher. Therefore, the full potential of Feynman diagrams, which includes the simplification of calculations involving the terms of perturbation series, has not been fully utilized. Feynman diagrams suggested in this study attempt to further the use of the notational power of Feynman diagrams in seismic applications. Instead of

\footnotetext{
This is because the reciprocity theorem of cross-correlation type involves both incoming and outgoing Green's functions in each of the two terms of the surface integral.

${ }^{2}$ Virtual particle is a term used in QFT to refer to a mode of the field that does not satisfy the energy-momentum relation. When the energy-momentum relation is satisfied that mode is said to be 'on mass shell' and it represents a real particle. Energy-momentum relation for Klein-Gordon field is $-|\mathbf{k}|^{2}+\omega^{2}=m^{2}$ (in natural units $c=1$ and $\hbar=1$ ). For zero mass $(m=0)$ this is just the dispersion relation for an acoustic field with velocity $=1$. Therefore, the corresponding virtual particles in the acoustic field are the modes which do not satisfy the dispersion relation.
} 
relying on inverting the Born series, GIMI relies on the stationary phase principal to cancel all but the optimal paths between scatterers and trial image points. Therefore, GIMI could come in the category of schemes which draw ideas from QFT.

The seismic scattering series and specifically interferometry involve cumbersome notation, especially in our case where we have to analyse higher order terms in the Born series. To make the derivations simpler and more intuitive, we introduce Feynman-like diagrams to represent different terms of the perturbation (Born) series. We shall also define some rules to represent operations, especially cross-correlation and convolution, on the terms of the perturbation series. Once Feynman-like diagrams and rules have been defined, we shall see that the theoretical derivations become very easy.

\section{FEYNMAN-LIKE DIAGRAMS}

Let us begin our development from the Lippmann-Schwinger equation that is given by (1) when both the source and receiver points are on the surface,

$G\left(\mathbf{y}^{\prime}, \mathbf{y}\right)=G_{0}\left(\mathbf{y}^{\prime}, \mathbf{y}\right)+\omega^{2} \int_{V} \delta v(\mathbf{x}) G\left(\mathbf{y}^{\prime}, \mathbf{x}\right) G_{0}(\mathbf{x}, \mathbf{y}) \mathrm{d}^{3} \mathbf{x}$,

where $G_{0}$ is the background Green's function, $G$ is the total Green's function and $\omega$ is the angular frequency. All the Green's functions in this study, for simplicity in presentation, are in frequency domain. $\delta v(\mathbf{x})$ is the slowness (reciprocal of medium velocity) perturbation. The Lippmann-Schwinger equation, with a source (or receiver) on the surface and a receiver (or source) inside the volume, is given as

$G(\mathbf{y}, \mathbf{x})=G_{0}(\mathbf{y}, \mathbf{x})+\omega^{2} \int_{V} \delta v\left(\mathbf{x}^{\prime}\right) G\left(\mathbf{y}, \mathbf{x}^{\prime}\right) G_{0}\left(\mathbf{x}, \mathbf{x}^{\prime}\right) \mathrm{d}^{3} \mathbf{x}^{\prime}$

The background Green's function, based on the cross-correlation interferometric theory, also satisfies the following relation Wapenaar \& Fokkema (2006):

$G_{0}^{*}\left(\mathbf{x}^{\prime}, \mathbf{x}\right)+G_{0}\left(\mathbf{x}^{\prime}, \mathbf{x}\right) \approx \int_{S} G_{0}^{*}(\mathbf{y}, \mathbf{x}) G_{0}\left(\mathbf{x}^{\prime}, \mathbf{y}\right) \mathrm{d}^{2} \mathbf{y}$.

In the Born scattering series from eq. (1), we cross-correlate with background Green's function and use (3). It is obvious that the notation then becomes very cumbersome. We therefore propose a different type of notation for these equations to represent these equations that is based on Feynman diagrams. Feynman diagrams have proved to be an invaluable tool to analyse the terms of the perturbation expansion for scattering processes in QFT. We shall use similar diagrams for the terms of the Born series for scattering processes in seismic imaging.

Using Feynman diagrams eq. (1) can be represented as

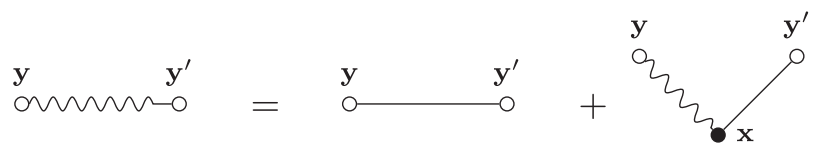

where a hollow circle denotes a point on the surface, a solid circle denotes a point inside the volume, a wiggly line represents the total Green's function and a solid line represents the background Green's function. Similarly, eqs (2) and (3) can be represented as

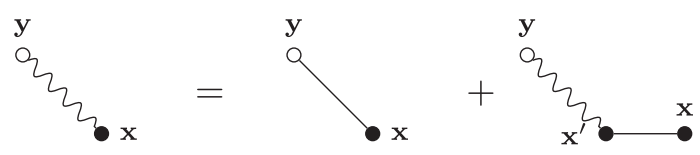

and

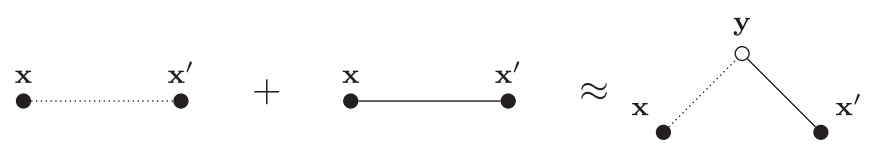

where the dotted straight line represents the complex conjugate of the background Green's function.

In eqs (4)-(6) endpoints and vertices were labelled to illustrate their meaning only, and from now onwards the endpoints will not be labelled. Labels on vertices will have a different meaning that will be defined with the rules for drawing Feynman diagrams. The wavefield is assumed to be acoustic. Along with the result from cross-correlation interferometry (eq. 3) we define the following rules to draw Feynman diagrams (Table 1).

The lines in the diagrams do not have directions marked by arrows because Green's functions are symmetric, and thus, it does not make a difference which direction we go. For convenience, source points will be considered to be on the left and receiver points on right, and surface points (hollow circles) will be drawn at the same level. Separation of vertices and endpoints just depicts different dummy variables for integration. Note that for surface seismic data the external lines will always end on the surface and loop diagrams do not have any external 
Table 1. Feynman rules.

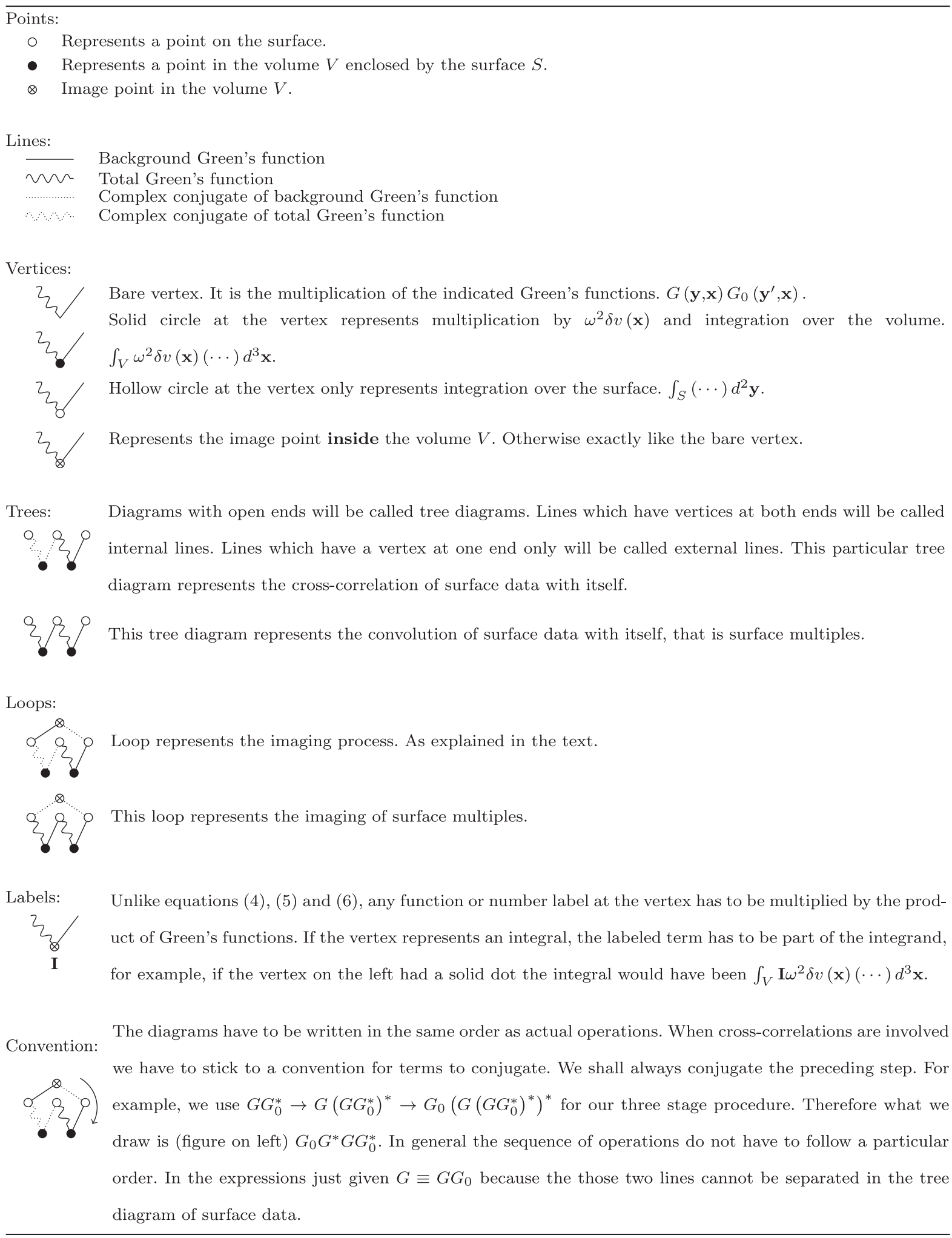

lines. Another important point to keep in mind is that when a solid (or hollow) circle is at a vertex, it represents integration over the volume (or surface). When a solid (or hollow) circle is at the free end of an external line, only then it represents a point inside the volume (or surface). Since in this study we are dealing with imaging and therefore loop diagrams, there are no external lines in the derivations which follow. This means that in all the following derivations the solid (or hollow) circles appear at vertices and hence represent an integration over the volume (or surface) as explained in the 'Vertices' section of Table 1. A comparison with the conventional algebraic representation is given in Appendix. 
Eq. (6) shows that a surface vertex can be removed when it represents a cross-correlation. This enables us to reduce our diagrams to those that do not contain any surface vertices. Such diagrams will be referred to as being in an irreducible form.

Before starting the derivations, it would not be out of place to mention a few other applications of these Feynman diagrams. One possible area of application, very closely related to the one described in this study, is the surface multiples. Surface multiples can be represented in terms a tree diagram. The second tree diagram shown in Table 1 represents first-order surface multiples, similarly further convolutions of surface data with itself can be drawn to represent higher orders. Furthermore, closing the loop in that tree diagram represents imaging of surface multiples as shown in the second loop diagram in Table 1.

Another possible application is to represent attenuation in the wavefields. Since attenuation only affects the amplitude and not the travelime of the wave, it could be handled by just labelling the endpoints or vertices in the diagram. Attenuation also implies dispersion and since these diagrams are per frequency, the labelling process is justified. To obtain the wavefield or image, the frequencies have to be summed and therefore the correct dispersion will be taken into account automatically. Note that the cross-correlation processes used in this study ignores amplitude by ignoring the gradient of one of the wavefields in the boundary integrals. Therefore, in order for attenuation to make sense the label has to include an appropriate gradient operator as well. Note that eq. (3) does not take attenuation into account, therefore the reduction operations on the diagrams would not be valid unless an equivalent expression which takes attenuation into account is used.

Feynman diagrams in this study just depict the Born series and anything implicit in it will be implicit in the diagrams too. Explicit representations of components such as head waves will require further study but it will take us beyond the scope of this paper.

\section{CONVENTIONAL RTM}

Now that we are done with the notation, let us look at the elementary process of seismic imaging, that is, imaging only single scattering. Single scattering can be imaged in various ways, one example is Kirchhoff migration. Reverse time migration (RTM) also images single scattering and if the velocity model is such that it does not produce any internal scatterings in the forward propagation step, this is its main contribution to the image.

Although direct arrivals can be of use in certain situations (Zuberi \& Alkhalifah 2013a), in conventional RTM direct arrivals are removed and we work only with the scattered part, which is the second term on the right-hand side (rhs) of eq. (1). The imaging process can be defined as closing the loop in a surface-to-surface diagram by cross-correlation at both ends by surface-to-volume background Green's functions, in which the point where they end in the volume is the image point. Summation over frequency of the final result is implied. If there was a scatterer at the image point in the subsurface the total phase around the loop would be zero and our zero-lag cross-correlation would give a non-zero amplitude at that point. The RTM can be represented as ${ }^{3}$

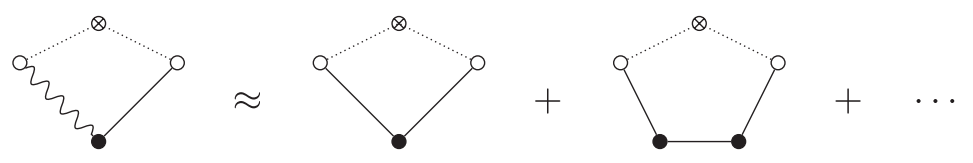

First term on the rhs in eq. (7) represents the imaging of single scattering. Its diagram represents

$\gamma^{\otimes} \equiv \iint G_{0}^{*}\left(\mathbf{x}_{i}, \mathbf{y}\right)\left[\int \omega^{2} G_{0}(\mathbf{x}, \mathbf{y}) \delta v(\mathbf{x}) G_{0}\left(\mathbf{y}^{\prime}, \mathbf{x}\right) d^{3} \mathbf{x}\right] G_{0}^{*}\left(\mathbf{x}_{i}, \mathbf{y}^{\prime}\right) d^{2} \mathbf{y} d^{2} \mathbf{y}^{\prime}$

In this study, we are interested in higher order scattering so let us look at the second term. Note that in the second term in eq. (7) vertices involving cross-correlation at the surface can be further reduced using eq. (3) as follows:

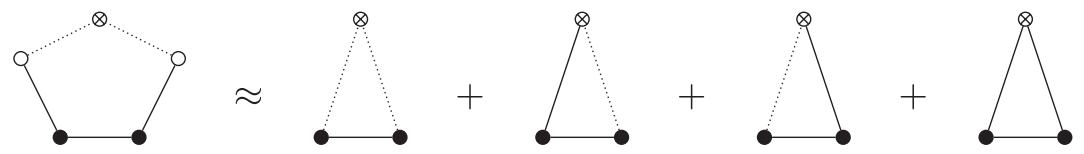

Now let us check the total phase of each of the four terms in the irreducible form. The fourth term on the rhs in eq. (9) will not give a zero phase at any image point. The first term on the other hand will have a zero phase when image point corresponds to either of the two vertices. ${ }^{4}$ The second term gives a zero phase when the image point corresponds to the first vertex and the third term when image point corresponds to the second vertex. It might also happen that either of the first three terms will give a zero phase at positions other than the vertices but only one term will contribute in that case, which will be weaker in amplitude than the actual scatterer position that gets contributions from two terms. Such noise can come from other higher order terms in eq. (7) (which we have not considered) as well, we shall refer to such noise as crosstalk. Also note that since volume vertices represent integration over the volume, it might happen that these two variables have the

\footnotetext{
${ }^{3}$ Note that eq. (7) not only represents RTM (with smooth velocity), it represents any imaging scheme that is based on single scattering assumption.

${ }^{4}$ Note that each vertex with a solid circle represents the total scatterer distribution and the line joining two vertices means scattering between different combinations of two scatterers from the aggregate. Therefore, each reduced form diagram is a sum of all such combinations.
} 
same position. In that case, eq. (9) will kinematically be equivalent to the single scattering image, but because we multiply with the square of perturbation term its amplitude will be lower.

Eq. (7) shows the first two terms of the infinite Born series. Obviously for the series to converge, the terms have to be smaller in magnitude as the order gets higher. In our diagrams each subsurface vertex (solid circle at the vertex) involves a multiplication by the perturbation term. Therefore, if the perturbation term is sufficiently small, the series will converge. This means that although the second term in RTM (eq. 7) images the second-order scattering, it's amplitude is much weaker than the first term.

In order to better understand the generalized procedure, it would be helpful to first look at the three-stage procedure recently published by Zuberi \& Alkhalifah (2013b), which is a special case for double scattering. We shall analyse it in terms of the Feynman-like diagrams.

\section{THE THREE-STAGE PROCEDURE (ZUBERI \& ALKHALIFAH 2013 b)}

The discussion above suggests that in order to image the double scattering (second-order term of the Born series or first-order internal multiples) in the subsurface we need to have the double scattering term either isolated or as the leading-order term in a series. To do this, we suggest a three-stage procedure.

(1) Back propagate the scattered field recorded on the surface to the imaging point in the volume.

(2) Cross-correlate the result of the first step with the recorded data again.

(3) Cross-correlate the result of the second stage with the surface-to-volume background Green's function (at the image point in the subsurface).

In diagram form (a comparison with the algebraic representation is given in Appendix) this can be represented as

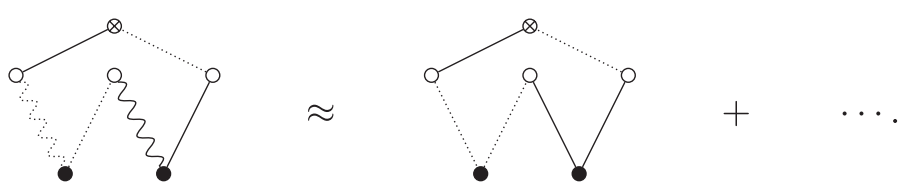

Eq. (10) shows the leading-order term after using eq. (2) for the total Green's functions. As discussed above we are interested only in the leading-order term. Note that this leading-order term for eq. (10) has two subsurface vertices. To check whether the scatterers will be imaged we need to write it in irreducible form. Since there are three surface vertices and each reduction according to eq. (3) will give two terms, we will have $8\left(2^{3}\right)$ irreducible terms for the leading order.

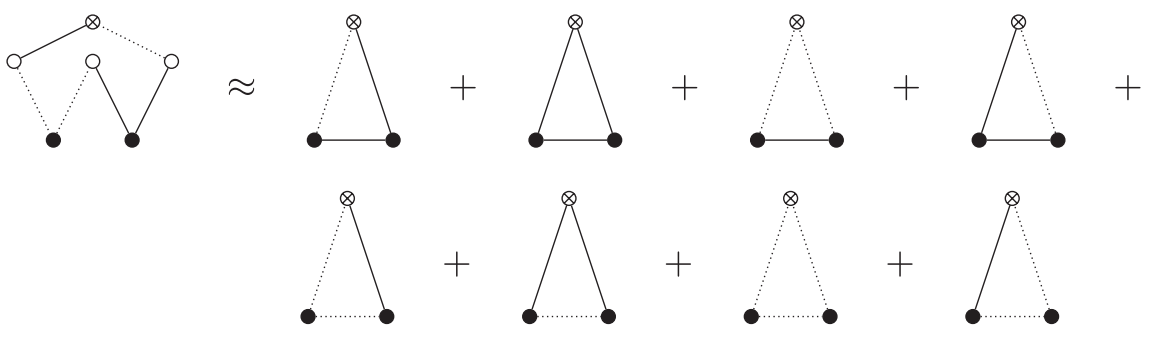

As before, when the image point corresponds to the left vertex, third, fourth, fifth and sixth terms contribute to the image. When the image point is on the right scatterer, first, third, sixth and eight terms contribute. Second and seventh term do not give a zero phase for any position of the image point. For the velocity model in our examples, we found that if after the first step, that is, back propagation of the data, we ignore the negative times, crosstalk noise is greatly reduced.

Note that four of the eight irreducible diagrams are complex conjugates of the other four. Therefore, there is a background zero phase that is like the low-frequency (long wavelength) noise in conventional RTM (not exactly the same). An application of the Laplacian filter at the end of imaging can easily get rid of it.

\section{G I M I}

From the analysis above we can find a general pattern for imaging higher-order internal multiples. First we note that in eq. (10), instead of the three stage procedure we can interpret the diagram on the lhs as

(i) Back propagate the data to the imaging point.

(ii) Cross-correlate with the data with itself $N-1$ times.

(iii) Cross-correlate the result with the background Green's function (from surface to image point).

Physically this means:

(i) Back propagate the receiver (or source) to the imaging point. 
(ii) Cross-correlate with the data with itself $N-1$ times.

(iii) Back propagate the source (or receiver) gathers.

Therefore, in general, to image the $N-1$ th order internal multiples we would have to cross-correlate the data $N-1$ times with itself and then apply the imaging condition. For example, the second-order internal multiple would be imaged like
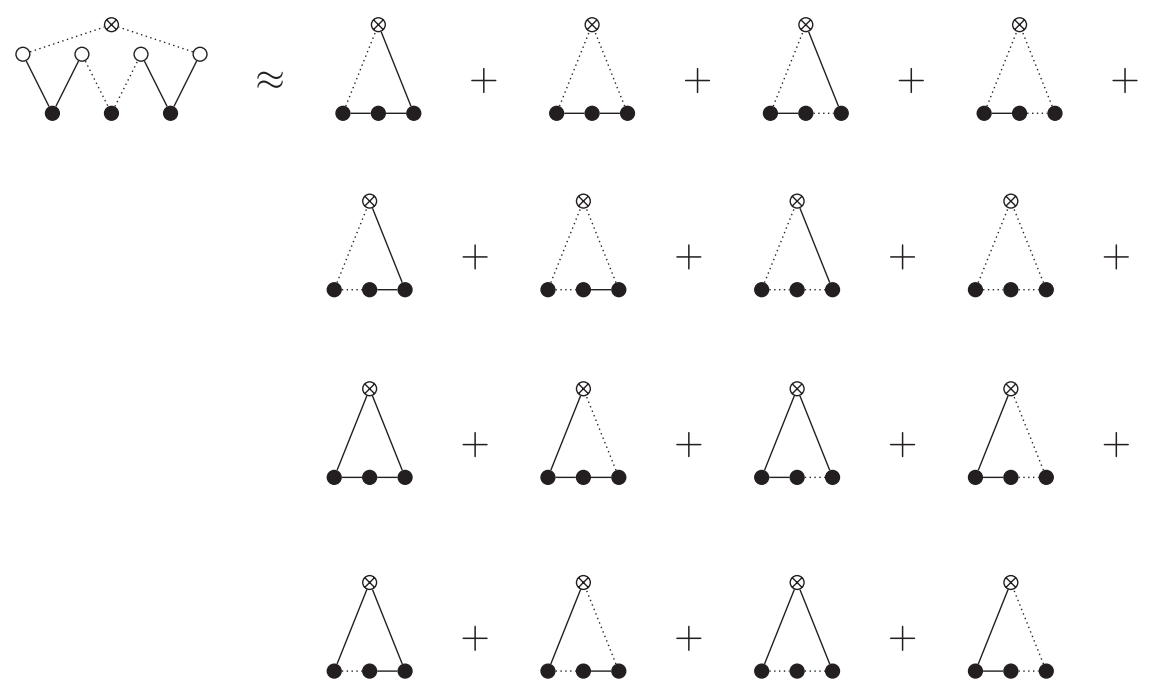

The left-hand side (lhs) of eq. (12) involves three volume vertices (third-order term in the Born series or second-order scattering) and four surface vertices. The four surface vertices will give $2^{4}$ terms in the irreducible form. In general for the $N$ th order term of the Born series we would have $N+1$ surface vertices and therefore $2^{N+1}$ terms in the irreducible form for the leading order term. Again we can find which terms in the irreducible form contribute to which imaging points. As there are more terms in the irreducible form there will also be more crosstalk, due to the zero phase at wrong imaging points. Although its amplitude relative to the correct image points (which gets contributions from more than one term) will be lower, it will appear in more places. We tackle this problem of crosstalk for the imaging of second-order scattering in the next section.

\section{CROSSTALK SUPPRESSION}

In eqs (10) and (11) crosstalk terms exist causing artifacts in the image of first-order multiples. The crosstalk is also present in the singlescattering image. Wong et al. (2012) suggested a method to suppress such noise. However, it will require a least-square formulation of the imaging process, in which we fit the modelled data to the recorded data. Since the fitting process requires that the amplitudes should be accurate, we, alternatively, rely on a dot product (zero-lag cross-correlation) of the modelled and recorded surface data. In this case, the initial image is used to model the scatterers Wong et al. (2012). For single scattering, the initial image I is multiplied by the back-propagated wavefield and convolved again with the background Green's function to the surface, as follows:
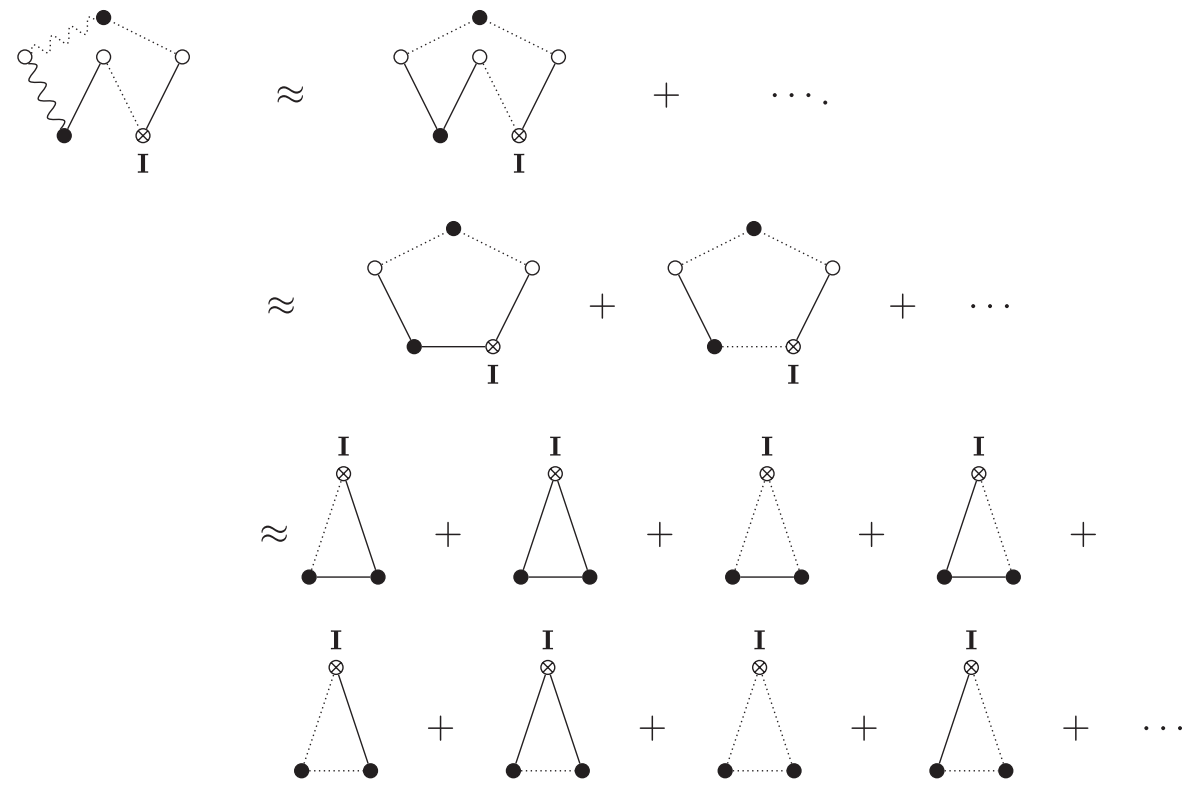
In eq. (13), the irreducible form is the same as in eq. (11) except for the multiplication with I. If I represents the second-order internal multiple image in eq. (11), then the right-hand side of eq. (13) is nothing but a product of eq. (11) with itself. This means we are just squaring the image.

\section{EXA MPLES}

We tested the approach on a simple model shown in Fig. 1. In this velocity model the vertical reflector at $1.3 \mathrm{~km}$ horizontal location will not be illuminated by the single-scattering recorded energy on the surface. We place a scatterer at $x=1.6 \mathrm{~km}$ and $z=0.36 \mathrm{~km}$. Dimensions of the scatterer are $40 \times 40 \mathrm{~m}$. Seismic survey for this synthetic experiment is a single 2-D line $3 \mathrm{~km}$ long, the velocity model and image figures have been zoomed to a region $x=2.5 \mathrm{~km}$ and $z=0.8 \mathrm{~km}$. Source and receiver intervals are $24 \mathrm{~m}$ each. In all the migrations for these examples total surface data have been used for imaging, direct arrivals have not been muted. Receiver spread for all the shots covers the whole line. Velocity of the reflecting surface and the scatterer is $3 \mathrm{~km} \mathrm{~s}^{-1}$ and that of the background is $2 \mathrm{~km} \mathrm{~s}^{-1}$. Fig. 2 shows how double scattering can help illuminate part of the vertical reflector. It is a sketch of ray paths coming from one shot. The sketch in Fig. 2 does not represent the Feynman-like diagrams, it just shows a single ray coming from one shot will scatter and then the scattered rays (shown in light grey) reflect from the vertical reflector before arriving at the receivers. Some of those doubly scattered waves will end up on the surface while others will not. Especially any reflections thar happen below the horizontal position of the scatterer will not end on the recording area. Fig. 3 shows the single scattering image where the vertical reflector has only been imaged at the surface, which is because of the direct arrivals (e.g. Zuberi \& Alkhalifah 2013a). Fig. 4 shows the double scattering image. It can been seen in this image that the vertical reflector has been imaged below the surface. In both Figs 3 and 4 there is a lot of noise. As discussed before we shall suppress the noise by squaring the image, the result is shown in Figs 5 and 6.

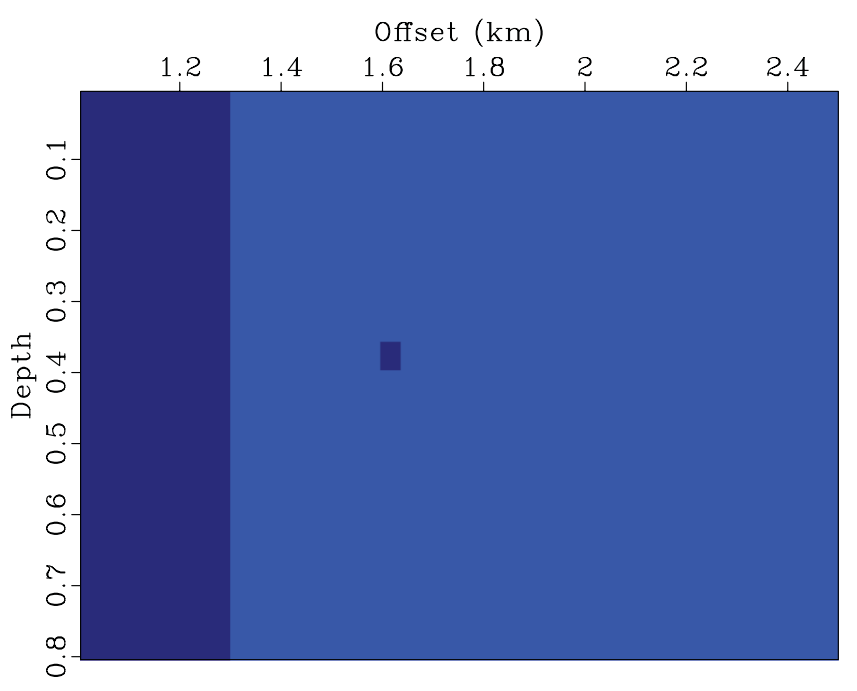

Figure 1. Velocity model.

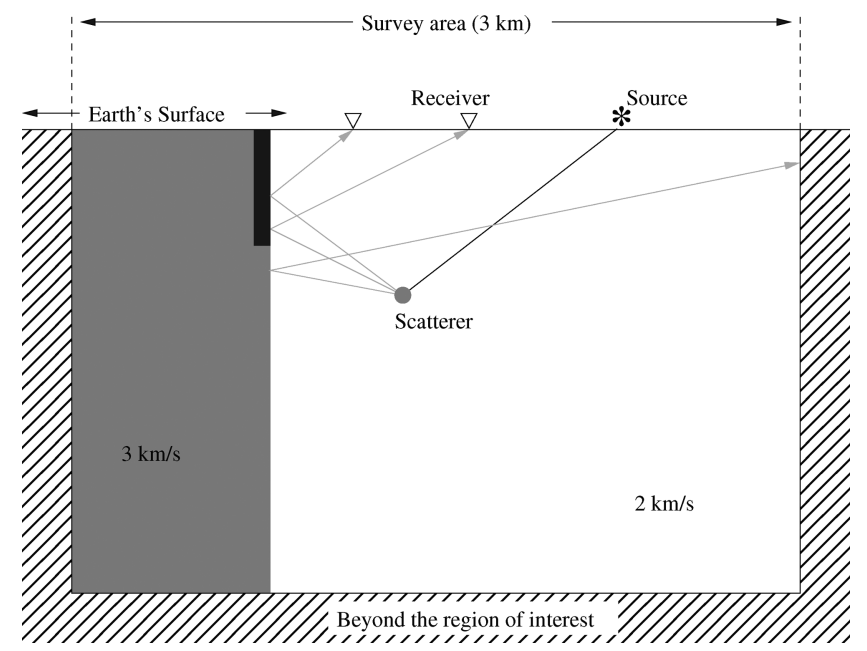

Figure 2. Sketch of ray paths from a source getting scattered by a scatterer. Black strip in the vertical reflector indicates approximately the portion which is most likely to be imaged by a surface seismic experiment. 


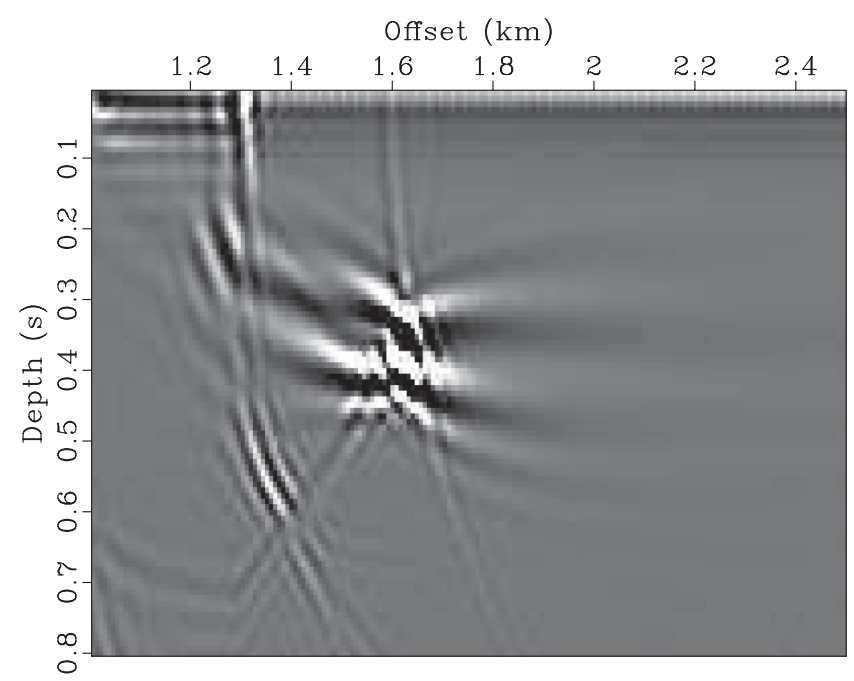

Figure 3. Single scattering image.

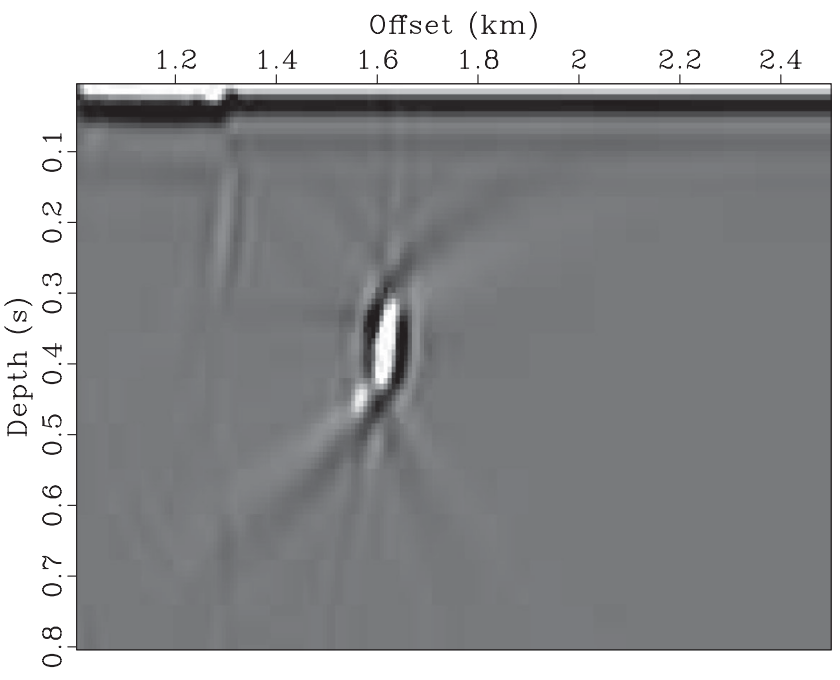

Figure 4. Double scattering image.

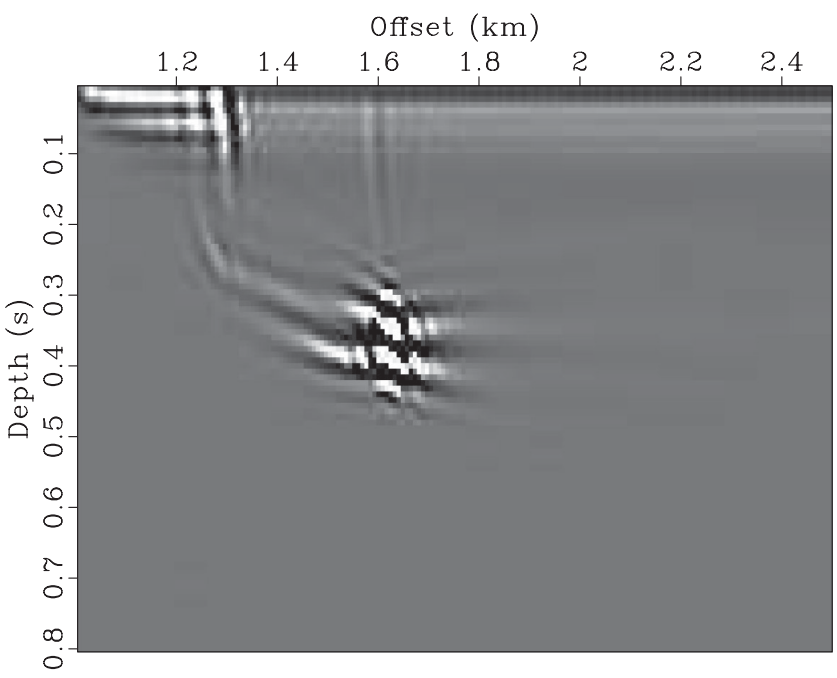

Figure 5. Single scattering image after crosstalk suppression. 


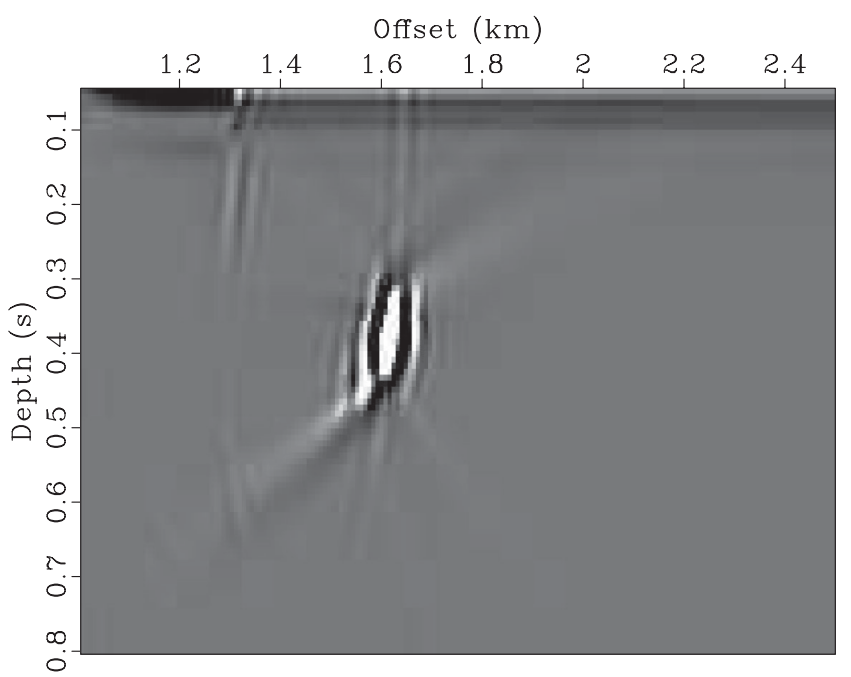

Figure 6. Double scattering image after crosstalk suppression.

\section{CONCLUSION}

We introduce GIMI as a process to image internal multiple, and we describe its mechanism, which can be complex, using Feynman-like diagrams to make the derivations easier and intuitively illuminating. The process allows imaging of any order of internal multiples separately and the cost is an additional cross-correlation step per multiple order. GIMI for the second-order term of the Born series (first-order internal multiples) is demonstrated on a simple velocity model with one vertical reflector and a scatterer beside it. The method used here to suppress the crosstalk in GIMI amounts to just squaring the image.

\section{ACKNOWLEDGEMENTS}

We thank the associate editor, Kees Wapenaar, and an anonymous reviewer for their critical and helpful review of the paper. We would like to thank King Abdullah University of Science and Technology (KAUST) for financial support. We would also like to thank members of Seismic Wave Analysis Group (SWAG).

\section{REFERENCES}

Aktosun, T. \& Rose, J.H., 2002. Wave focusing on the line, J. Math. Phys., 43(7), 3717-3745.

Behura, J., Wapenaar, K. \& Snieder, R., 2012. Newton-Marchenko-Rose Imaging, Chap. 685, pp. 1-6, SEG.

Berkhout, A.J. \& Verschuur, D.J., 1994. Multiple technology: part 2, migration of multiple reflections, SEG Expanded Abstracts, 13, 14971500 .

Broggini, F. \& Snieder, R., 2012. Connection of scattering principles: a visual and mathematical tour, Eur. J. Phys., 33(3), 593.

Chadan, K. \& Sabatier, P.C., 1977. Inverse Problems in Quantum Scattering Theory, Springer-Verlag.

Fishman, L., McCoy, J.J. \& Wales, S.C., 1987. Factorization and path integration of the helmholtz equation: numerical algorithms, J. acoust. Soc. Am., 81(5), 1355-1376.

Ikelle, L.T. \& Gangi, A.F., 2007. Negative bending in seismic reflection associated with time-advanced and time-retarded fields, Geophys. Prospect., 55(1), 57-69.

Jost, R. \& Kohn, W., 1952. Construction of a potential from a phase shift, Phys. Rev., 87, 977-992.

Landa, E., Fomel, S. \& Moser, T., 2006. Path-integral seismic imaging, Geophys. Prospect., 54(5), 491-503.

Malcolm, A.E., Ursin, B. \& De Hoop, M.V., 2009. Seismic imaging and illumination with internal multiples, Geophys. J. Int., 176(3), 847864
Rose, J.H., 2004. Single-sided focusing and the minimum principle of inverse scattering theory, Inverse Probl., 20(1), 243.

Sakurai, J.J., 1993. Modern Quantum Mechanics (Revised Edition), 1st edn, Addison Wesley.

Shan, G., 2003. Source-receiver migration of multiple reflections, SEG Expanded Abstracts, 22, 1008-1011.

Verschuur, D., Berkhout, A. \& Wapenaar, C., 1992. Adaptive surface-related multiple elimination, Geophysics, 57(9), 1166-1177.

Wapenaar, K. \& Fokkema, J., 2006. Green's function representations for seismic interferometry, Geophysics, 71(4), SI33-SI46.

Wapenaar, K., Broggini, F. \& Snieder, R., 2012. Creating a virtual source inside a medium from reflection data: heuristic derivation and stationaryphase analysis, Geophys. J. Int., 190(2), 1020-1024.

Weglein, A.B. et al., 2003. Inverse scattering series and seismic exploration, Inverse Probl., 19(6), R27-R83.

Wong, M., Biondi, B. \& Ronen, S., 2012. Imaging with Multiples using Linearized Full-Wave Inversion, Chap. 706, pp. 1-5, SEG.

Zee, A., 2003. Quantum Field Theory in a Nutshell, illustrated edn, Princeton Univ. Press.

Zuberi, A. \& Alkhalifah, T., 2013a. Imaging by forward propagating the data: theory and application, Geophys. Prospect., 61 (60 Year Anniversary Issue), 248-267.

Zuberi, M.A.H. \& Alkhalifah, T., 2013b. Selective interferometric imaging of internal multiples, EAGE Extended Abstract, 75th EAGE Conference \& Exhibition Incorporating SPE EUROPEC 2013, EAGE. 


\section{APPENDIX A: COMPARISON WITH ALGEBRAIC REPRESENTATION}
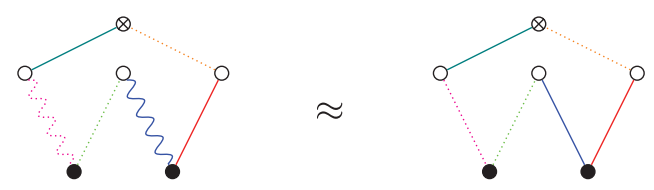

(A1a)

$$
\begin{gathered}
\omega^{4} \int_{V} \int_{V} \int_{S} \int_{S} \int_{S} \delta v\left(\mathbf{x}_{2}\right) \delta v\left(\mathbf{x}_{4}\right) G_{0}\left(\mathbf{x}_{i}, \mathbf{x}_{5}\right) G^{*}\left(\mathbf{x}_{5}, \mathbf{x}_{4}\right) G_{0}^{*}\left(\mathbf{x}_{4}, \mathbf{x}_{3}\right) \\
G\left(\mathbf{x}_{3}, \mathbf{x}_{2}\right) G_{0}\left(\mathbf{x}_{2}, \mathbf{x}_{1}\right) G_{0}^{*}\left(\mathbf{x}_{i}, \mathbf{x}_{1}\right) \mathrm{d}^{2} \mathbf{x}_{5} \mathrm{~d}^{2} \mathbf{x}_{3} \mathrm{~d}^{2} \mathbf{x}_{1} \mathrm{~d}^{3} \mathbf{x}_{4} \mathrm{~d}^{3} \mathbf{x}_{2} \approx \\
\omega^{4} \int_{V} \int_{V} \int_{S} \int_{S} \int_{S} \delta v\left(\mathbf{x}_{2}\right) \delta v\left(\mathbf{x}_{4}\right) G_{0}\left(\mathbf{x}_{i}, \mathbf{x}_{5}\right) G_{0}^{*}\left(\mathbf{x}_{5}, \mathbf{x}_{4}\right) G_{0}^{*}\left(\mathbf{x}_{4}, \mathbf{x}_{3}\right) \\
G_{0}\left(\mathbf{x}_{3}, \mathbf{x}_{2}\right) G_{0}\left(\mathbf{x}_{2}, \mathbf{x}_{1}\right) G_{0}^{*}\left(\mathbf{x}_{i}, \mathbf{x}_{1}\right) \mathrm{d}^{2} \mathbf{x}_{5} \mathrm{~d}^{2} \mathbf{x}_{3} \mathrm{~d}^{2} \mathbf{x}_{1} \mathrm{~d}^{3} \mathbf{x}_{4} \mathrm{~d}^{3} \mathbf{x}_{2}+\cdots
\end{gathered}
$$

Eq. (A1b) is the algebraic form of eq. (A1a). The G's in eq. (A1b) corresponding to the lines in eq. (A1a) can be identified by the colour coding. ${ }^{5}$ If we perform the surface integrals shown on the rhs of eq. (A1b) over $\mathbf{x}_{5}$ and $\mathbf{x}_{3}$ and use eq. (3), one of the terms we get (after rearranging the integrals) is

$\int_{S}\left[\omega^{4} \int_{V} \int_{V} G_{0}\left(\mathbf{x}_{i}, \mathbf{x}_{4}\right) \delta v\left(\mathbf{x}_{4}\right) G_{0}\left(\mathbf{x}_{4}, \mathbf{x}_{2}\right) \delta v\left(\mathbf{x}_{2}\right) G_{0}\left(\mathbf{x}_{2}, \mathbf{x}_{1}\right) \mathrm{d}^{3} \mathbf{x}_{4} \mathrm{~d}^{3} \mathbf{x}_{2}\right] G_{0}^{*}\left(\mathbf{x}_{i}, \mathbf{x}_{1}\right) \mathrm{d}^{2} \mathbf{x}_{1}$.

The term in brackets in eq. (A2) is the second-order term of the Born scattering series, which represents double scattering inside the volume. Again using eq. (3) to perform the remaining surface integral in eq. (A2), one of the terms we get is

$\omega^{4} \int_{V} \int_{V} \delta v\left(\mathbf{x}_{4}\right) \delta v\left(\mathbf{x}_{2}\right) G_{0}\left(\mathbf{x}_{i}, \mathbf{x}_{4}\right) G_{0}\left(\mathbf{x}_{4}, \mathbf{x}_{2}\right) G_{0}^{*}\left(\mathbf{x}_{i}, \mathbf{x}_{2}\right) \mathrm{d}^{3} \mathbf{x}_{4} \mathrm{~d}^{3} \mathbf{x}_{2}$

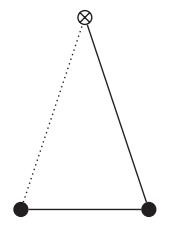

(A3b)

In the term shown in eq. (A3a) when $\mathbf{x}_{i} \equiv \mathbf{x}_{4}$, the total phase is zero. Therefore, when the frequencies are summed (to apply the zero-time imaging condition) this term will contribute to the image. Note that each application of eq. (3) gives two terms, one involving $G$ and the other $G^{*}$. In obtaining the term shown in (A2) we picked only $G$ 's and for (A3) only $G^{*}$. There are other terms, corresponding to different choices of $G$ 's. The diagram form of (A3a), shown in (A3b) is just the first term of the full eight shown in eq. (11) in the text.

\footnotetext{
${ }^{5}$ The colour coding is used here only for illustration, it is not a part of the diagram representation.
} 